\title{
Mixed-State Models for Nonstationary Multiobject Activities
}

\author{
Naresh P. Cuntoor and Rama Chellappa \\ Department of Electrical and Computer Engineering, Center for Automation Research, University of Maryland, A. V. Williams Building, \\ College Park, MD 20742, USA
}

Received 13 June 2006; Revised 20 October 2006; Accepted 30 October 2006

Recommended by Francesco G. B. De Natale

We present a mixed-state space approach for modeling and segmenting human activities. The discrete-valued component of the mixed state represents higher-level behavior while the continuous state models the dynamics within behavioral segments. A basis of behaviors based on generic properties of motion trajectories is chosen to characterize segments of activities. A Viterbi-based algorithm to detect boundaries between segments is described. The usefulness of the proposed approach for temporal segmentation and anomaly detection is illustrated using the TSA airport tarmac surveillance dataset, the bank monitoring dataset, and the UCF database of human actions.

Copyright (c) 2007 N.P. Cuntoor and R. Chellappa. This is an open access article distributed under the Creative Commons Attribution License, which permits unrestricted use, distribution, and reproduction in any medium, provided the original work is properly cited.

\section{INTRODUCTION}

Modeling complex activities involves extracting spatiotemporal descriptors associated with objects moving in a scene. It is natural to think of activities as a sequence of segments in which each segment possesses coherent motion properties. There exists a hierarchical relationship extending from observed features to higher-level behaviors of moving objects. Features such as motion trajectories and optical flow are continuous-valued variables, whereas behaviors such as start/stop, split/merge, and move along a straight line are discrete-valued. Mixed-state models provide a way to encapsulate both continuous and discrete-valued states.

In general, the activity structure, that is, the number of behaviors and their sequence, may not be known a priori. It requires an activity model that cannot only adapt to changing behaviors but also one that can learn incrementally and "on the fly." Many existing approaches assume that the structure of activities is known; and a fixed number of free parameters is determined based on experience or by estimating the model order. The structure then remains fixed. This may be a reasonable assumption for activities such as walking and running, but becomes a serious limitation when modeling complex activities in surveillance and other scenarios. We are interested in these classes of activities. Instead of assuming a fixed global model order, local complexity is constrained using dynamical primitives within short-time seg- ments. We choose a basis of behaviors that reflects generic motion properties to model these primitives. For example, the basis elements represent motion with constant velocity along a straight line, curved motion, and so forth. Using the basis of behaviors, we present two behavior-driven mixedstate (BMS) models to represent activities: offline and online BMS models. The models are capable of handling multiple objects, and the number of objects in the scene may vary with time. The basis elements are not specific to a particular video sequence, and can be used to model similar scenarios.

We present a Viterbi-based algorithm to estimate the switching times between behaviors and demonstrate the usefulness of the proposed models for temporal segmentation and anomaly detection. Temporal segmentation is useful for indexing and easy storage of video sequences, especially in surveillance videos where a large amount of data is available. Besides the inherent interest in detecting anomalies in video sequences, anomaly detection may also provide cues about important information contained in activities.

The rest of the paper is organized as follows. Section 2 describes low-level processing methods for detecting and tracking moving objects. The kinematics of extracted trajectories is modeled using linear systems. Section 3 describes offline and online BMS models. Section 4 describes a basis for representing segments of video sequences and a Viterbi-based algorithm for segmentation. Section 5 illustrates the usefulness of the proposed method using temporal segmentation 
and anomaly detection. The airport surveillance TSA dataset, the bank surveillance dataset, and the UCF database of human actions are used. Section 6 concludes the paper.

\section{Remark on notation and terminology}

We use the term nonstationary activities to suggest that parameters of behavior can change with time. The term has been used in similar contexts in both speech [1] and activity recognition [2].

Throughout the paper, we use $x(t) \in R^{n}$ to represent a continuous-valued variable and $q(t) \in\{1,2, \ldots, N\}$ to represent a discrete-valued variable. We use the notation $x_{t_{1}}^{t_{2}}$ to denote the sequence $\left\{x\left(t_{1}\right), x\left(t_{1}+1\right), \ldots, x\left(t_{2}\right)\right\}$.

\subsection{Related work}

For more than a decade, activity modeling and recognition has been an active area of research. Several methods have been proposed to represent and recognize simple activities such as walking, running, hopping, and so forth (see $[3,4]$ ). Aggarwal and Cai [3] present a comprehensive review of human motion and activities. They classify human activity recognition algorithm into two groups: state-space and template matching approaches (see $[5,6])$. State-space models have been applied in many problems ranging from gesture $($ see $[4,7])$ to gait $($ see $[8,9])$ to complex activities (see $[10])$.

\subsubsection{Event-and primitive-based models}

Approaches to modeling complex activities can be broadly divided into two groups: those based on events and those based on primitives. Events are based on certain instantaneous changes in motion while primitives are based on dominant properties of segments. Nevatia et al. [11] present a formal language for modeling activities. They define an event representation language (ERL) that uses an underlying ontological structure to encode activities. Syeda-Mahmood et al. [12] use generalized cylinders to represent actions. Assuming that the start and end points are known, they formulate the task as a joint action recognition and fundamental matrix recovery problem. Rao et al. [13] represent actions using dynamic instants, which are points of maximum curvature along the trajectory. Event-based representations are best suited when sufficient domain knowledge and robust low-level algorithms that can distinguish between noisy spikes and spikes due to instantaneous events are available. Ivanov and Bobick [7] use the outputs of primitive HMMs along with stochastic context-free grammar to parse activities with known structure. Coupled HMMs have been used in [10] for complex action recognition. Koller and Lerner [14] described a sampling approach for learning parameters of a dynamic Bayesian network (DBN). Hamid et al. [15] use the DBN framework for tracking complex activities assuming that the structure of the graph is fixed and known. Vu et al. [16] present an activity recognition framework that combines subscenarios and associated spatiotemporal and logical constraints.

\subsubsection{Mixed-state models}

Mixed-state models have been used for several applications including activity modeling, air traffic management, smart highway system, and so forth (see [17-20]). In some of these applications such as $[19,20]$, the focus is on analyzing the mixed-state systems where the model parameters are known (by design). On the other hand, like $[17,18]$, we are interested in learning parameters of mixed-state models. Unlike HMMs, parameter estimation in mixed-state models is intractable. Isard and Blake present a sampling technique for estimating a mixed-state model [17]. They assume that the structure of the activities is known, and that the parameters are stationary. Ghahramani and Hinton describe a variational method for learning [18].

\subsubsection{Activity recognition and anomaly detection}

An unsupervised system for classification of activities was developed by Stauffer and Grimson [21]. Motion trajectories collected over a long period of time were quantized into a set of prototypes representing the location, velocity, and object size. Parameswaran and Chellappa [22] compute view invariant representations for human actions in both $2 \mathrm{D}$ and $3 \mathrm{D}$. In $3 \mathrm{D}$, actions are represented as curves in an invariance space and the cross ratio is used to find the invariants. Vaswani et al. [2] model a sequence of moving points engaged in an activity using Kendall's shape space theory [23]. In situations where the activity structure is known, Zhong et al. [24] propose a similarity-based approach for detecting unusual activities.

It may be useful to compare the proposed models with the HMM approach and other mixed-state models in order to place our work in context. The HMM topology, that is, the number of states and the structure of the transition matrix is assumed to be known. The state transitions are assumed to be Markovian. The observed data is assumed to be conditionally independent of its past given the current hidden state. Also, the output distribution is assumed to be stationary. This makes the estimation procedure tractable. The Viterbi algorithm is then used to find the optimal state sequence efficiently.

We address some of these issues in the proposed activity model. In particular, the evolution of hidden (discrete) states is allowed to depend on the continuous state, which relaxes the Markov assumption. This causes the computational complexity of the parameter estimation process to grow exponentially [18]. To overcome this problem, we introduce a basis of behaviors motivated by motion properties of typical activities of humans and vehicles within a short-time window. A basis can be chosen so that it applies to similar scenarios across datasets. In our experiments, the same basis of behaviors is used in both the TSA airport surveillance dataset and the bank monitoring dataset. Further, we present a cost-based Viterbi algorithm instead of the usual probability-based one, since it is not easy to compute the normalization terms of the probability distribution. 


\section{LOW-LEVEL VIDEO PROCESSING}

The types of activity of interest may be illustrated using the following example. In video sequences of an airport tarmac surveillance scenario, we may observe segments of activities such as movement of ground crew personnel, arrival and departure of planes, movement of luggage carts to and from the plane, and embarkation and disembarkation of passengers. The video sequences are usually long. It would be useful to segment and recognize activities for convenient storage and browsing. Viewed as an inference problem, activity modeling involves learning parameters of behaviors using motion trajectories extracted from video sequences.

Motion trajectories and apparent velocities are continuous-valued variables that can be modeled using statespace models. In this section, a brief outline of low-level procedures to extract motion trajectories is described and a way of handling multiple objects is presented.

\subsection{Detection and tracking}

Tracking is challenging in surveillance scenarios due to low video resolution, low contrast, and noise. Instead of attempting to track objects across the entire video sequence, we periodically reinitialize the tracker. The low-level tasks may be divided into two components: moving object detection and tracking. The detection component uses background subtraction to isolate the moving blobs. We use a procedure based on $[25,26]$. The background in each RGB color channel is modeled using single independent Gaussian distributions at every pixel using ten consecutive frames. Frames in the video sequence are compared with the background model to detect moving objects. If the normalized Euclidean distance between the background model and the observed pixel value in a frame exceeds a certain threshold, then the pixel is labeled as belonging to a moving object. A static background is insufficient to model a long video sequence because of changing lighting conditions, shadows, and cumulative effects of noise. So the background is reinitialized at regular intervals.

Motion trajectories are obtained using the KLT algorithm [27] whose feature points are initialized at detected locations of motion blobs. The KLT algorithm selects features with high intensity variation and keeps track of these features. It defines a measure of dissimilarity to quantify the change in appearance between frames, allowing for affine image changes. Parameters control the maximum allowable interframe displacement and proximity of feature points to be tracked. The trajectories from the KLT tracker are smoothed using a median filter. The effect of tracking errors is discussed in Section 5. Of the three datasets used in the experiments, tracking was accurate and reliable in the indoor bank monitoring dataset and the UCF human action dataset. On the other hand, there were a few tracking errors in the TSA airport tarmac surveillance dataset that caused errors in temporal segmentation.

In the case of a single object moving in the scene, its motion trajectory and velocity (computed using finite differ- ences) forms the continuous-valued state $\{x(t), t \in[0, T]\}$, where $x(t) \in \mathcal{R}^{4}$. When several objects are present in the scene, this can be extended in a relatively straightforward manner if the number of objects remains constant. If the number of objects varies with time, there are several ways of defining the continuous state as described in the next section.

\subsection{Handling multiple objects}

Let $m(t)$ be the number of objects present in the scene at time $t$. Let $X_{c}(t) \in \mathcal{R}^{4 m(t)}$ represent the composite object. We use the notation $\mathbf{X}_{\mathbf{c}}(t)$ to indicate the sequence $\left\{X_{c}(1), X_{c}(2), \ldots, X_{c}(t)\right\}$. Each of the $m$ trajectories is associated with the observation sequence with four components representing the $2-\mathrm{D}$ position and velocity. Clearly, the number of objects $m(t)$ need not be constant. This problem of varying dimension can be handled in several ways. For example, $m(t)$ can be suitably augmented to yield a constant number $M$ by creating virtual objects. In [2], motion trajectories are represented using Kendall's shape space. The trajectory is resampled so that the shape is defined by $k$ points. As an illustration, consider the trajectory formed by passengers (treated as point objects) exiting an aircraft on a tarmac and walking toward the gate. The number of passengers in the scene $m(t)$ can vary with time. Irrespective of the value of $m(t)$, a common motion trajectory can be formed by connecting the position of the first passenger to that of the last passenger such that the curve passes through every passenger in the scene. The common trajectory is resampled at $k$ points creating $k$ virtual passenger positions, and used to represent the shape. This is equivalent to defining an abstracting map from a $4 m(t)$ $\mathrm{D}$ space to a $4 k-\mathrm{D}$ space. When the objects are not interacting or the nature of interaction is unknown, it is not clear how to place the $k$ virtual objects to obtain a constant cardinality.

Though there may be several objects in the scene, there are only a few types of activities. For instance, in a surveillance scenario, there may be several persons walking on a street. Each person has his/her own dynamics whose parameters can vary. Walking activity, however, is common across persons. This motivates the usefulness of constructing a basis of behavior. In this example, the direction and speed of walking could distinguish different basis elements.

The choice of a basis of behavior depends on the domain of application, but need not be specific to datasets. In our experiments, we use the same basis across two surveillance scenarios, one captured on airport tarmac and the other inside a bank. If there is insufficient domain knowledge to guide the selection of a basis, a generic basis based on eigenvalues of the system matrix can be used to distinguish between basis elements (Section 3.3).

The dynamics of objects in the scene is modeled individually using the most likely basis element. The number of objects $m(t)$ is allowed to vary at discrete time intevals so that $m(t)$ is constant over a short video segment. The change in the value of $m(t)$ is modeled as a one-step random walk. The conditional probability distribution function 
(pdf) for a segment $s$ can be written as $f\left(\mathbf{X}_{\mathbf{c}}(t), m(t) \mid S=\right.$ $s)=b_{s, m}\left(\mathbf{X}_{\mathbf{c}}(t)\right) P(m(t)=m \mid S=s)$. A behavior segment $s \in \mathcal{S}$ is characterized by the distribution of the number of objects in the scene $P(m \mid s)$ and a family of distributions $b_{s, m}\left(\mathbf{X}_{\mathbf{c}}(t)\right)$ that describes the segment. The pdf $b_{s, m}\left(\mathbf{X}_{\mathbf{c}}(t)\right)$ is calculated using a basis of behaviors. This value is used for temporal segmentation (Section 4.1). To place this definition in context, consider an HMM. In this case, the probability of the segment is written as the product $b_{s, m}\left(\mathbf{X}_{\mathbf{c}}(t)\right)=$ $\prod_{i=1}^{t} f\left(X_{c}(i) \mid s\right)$ and the HMM persists in this state with a geometric distribution.

\section{MIXED-STATE MODELS}

Let the sequence of discrete states be $\{q(1), q(2), \ldots, q(T)\}$, where $q(i) \in\{1,2, \ldots, N\}$ indexes the discrete-valued behavior. The objects may transit through $M$ behaviors, switching at time instants $\tau=\left\{\tau_{0}, \tau_{1}, \ldots, \tau_{M}\right\}$, where $\tau_{0}=0, \tau_{M}=T$. In general, the number of behaviors $M$ and switching instants $\tau_{i}$ 's are unknown. We present two BMS models to represent the behavior within such segments: offline and online BMS models, respectively.

Consider the general state equations of continuous and discrete variables:

$$
\begin{gathered}
\dot{x}(t)=h_{q(t)}(x(t), u(t)), \quad x(0)=x_{0}, \\
q^{+}(t)=g\left(q_{1}^{t-1}, x_{1}^{t-1}, n(t)\right) .
\end{gathered}
$$

The continuous state dynamics $h_{q(t)}$ depends on the discrete state $q(t)$. It captures the notion that a higher-level behavior evolves in time and generates correlated continuous-valued states $x(t)$. The continuous state dynamics within each segment is limited by the form of $h_{q(t)}$. The discrete state $q(t)$ evolves according to $g(\cdot)$ and depends not only on the previous discrete state, but also on past values of the observed data $x_{1}^{t-1} \cdot u(t)$, and $n(t)$ represent noise. This makes the evolution of discrete state non-Markovian. We make the following assumptions.

(A1) The number of discrete state switching times is finite.

(A2) Discrete state transitions occur at discrete time instants, that is, $\tau_{i}=k \alpha$ for $i=1, \ldots, M-1$, where $k, \alpha$ are integers.

(A3) Between consecutive switching instants $\tau_{i}, \tau_{i+1}, i=$ $1, \ldots, M$, the parameters of the continuous dynamical model do not change.

(A1) ensures that we do not run into pathological conditions such as Zeno behavior. ${ }^{1}$ (A2) and (A3) are the practical conditions required for robust estimation of parameters of each segment. We arrive at the offline and online BMS models by making certain additional assumptions in (1) and (2) as explained in Sections 3.2 and 3.3.

\footnotetext{
${ }^{1}$ Roughly speaking, an execution of a mixed system is called Zeno, if it takes infinitely many discrete transitions in a finite time interval.
}

\subsection{Special case: AR-HMM}

Before describing the proposed mixed-state models, we review the autoregressive (AR) HMM, which is a special case of (1) and (2). The AR-HMM was introduced in [28] using a cross entropy setting. In addition to (A1)-(A3), the ARHMM requires the following assumptions.

(A4) The number of discrete states $N$ is known.

(A5) The processes are stationary and the model parameters do not depend on time.

Similar to the HMM, the hidden state in the AR-HMM follows the Markov dynamics,

$$
P\left(q(t) \mid q_{1}^{t-1}, x_{1}^{t-1}\right)=P(q(t) \mid q(t-1)) .
$$

The joint distribution of the continuous and discrete states can be written as follows,

$$
\begin{aligned}
& f\left(x(t), q(t) \mid x_{1}^{t-1}, q_{1}^{t-1}\right) \\
& \quad=f\left(x(t), q(t) \mid q(t-1), x_{t-\alpha-1}^{t-1}\right) .
\end{aligned}
$$

This is useful for obtaining the optimal-state sequence using the Viterbi algorithm. Using (3) and (4), we have

$$
\begin{aligned}
& f\left(x(t), q(t) \mid q(t-1), x_{t-\alpha-1}^{t-1}\right) \\
& \quad=f\left(x(t) \mid q(t), x_{t-\alpha-1}^{t-1}\right) \times P(q(t) \mid q(t-1)) .
\end{aligned}
$$

The distribution $f(x \mid \cdot, \cdot)$ is assumed to be normal. The mean and variance depends on the discrete state. The parameters can be estimated using these hypotheses in an EM setting [29].

\subsection{Offline BMS model}

The Markov assumption of discrete state evolution in (3) means that the behavior parameters change without a direct dependence on the observed data. It would be more reasonable to allow past values of observed data to influence changes in behavior. So we present an offline BMS model whose discrete state transition is given by the following:

$$
f\left(q(t) \mid q_{1}^{t-1}, x_{1}^{t-1}\right)=f\left(q(t) \mid q(t-1), x_{t-\beta}^{t-\alpha}\right),
$$

where $q(t) \in\{1, \ldots, N\}$ for some known number of states $N$ and $\beta=k \alpha$ for some integer $k$. Let the effective state be $r(t)=\left(q(t), x_{t-\beta}^{t-\alpha}\right)$ so that (6) can be rewritten. The state evolution of $r(t)$ is Markov and the parameters and switching times can be computed, in principle, using algorithms similar to the AR-HMM case. The computation of the parameters, however, is not as elegant as the classical HMM and it is difficult to construct a recursive estimation procedure like the EM algorithm (briefly described in Section 4). Also, the transition probability $P(r(t) \mid r(t-1))$ depends on the observed data and violates assumption (A5). The transition 
probability of the effective state can be written as follows:

$$
\begin{aligned}
& f(r(t) \mid r(t-1))=f\left(x_{t-\beta}^{t-\alpha} \mid q(t), q(t-1), x_{t-\beta}^{t-\alpha}\right), \\
& f\left(q(t) \mid q(t-1), x_{t-\beta}^{t-\alpha}\right) \\
& =\frac{f\left(r(t-1) \mid q(t), q(t-1), x_{t-\beta}^{t-\alpha}\right)}{f\left(x_{t-\beta}^{t-\alpha} \mid q(t-1)\right)} \\
& \quad \times\left(f\left(x_{t-\beta}^{t-\alpha} \mid q(t), q(t-1)\right) \times f(q(t) \mid q(t-1))\right) .
\end{aligned}
$$

The probability in (7) is difficult to compute due to two main reasons. Unlike (3), (7) depends on $x_{t-\beta}^{t-\alpha}$. So the transition probability matrix is no longer stationary. For parameter estimation using the EM algorithm, the denominator term in (8) cannot be computed. So we turn to the underlying state (1), and define an offline BMS model as a sequence of linear dynamics. The calculation of probabilities can be replaced with running and switching costs incurred due to the estimated dynamical parameters. In addition to (A1)-(A4), we assume the following.

(A6) The segment-wise dynamics are linear, that is, (1) takes the following form:

$$
\dot{x}(t)=A_{q(t)} x(t)+b, \quad x(0)=x_{0},
$$

where $A_{q(t)} \in\left\{A_{1}, A_{2}, \ldots, A_{N}\right\}$ for some known $N$ are obtained by training.

The offline BMS model can be used for activity recognition and anomaly detection. Using training data, we can compute the parameters of normal behaviors. This allows us to not only check for anomalies but provide a way to localize anomalous parts of the activity, that is, the unexpected $A_{q(t)}$ segments.

\subsection{Online BMS model}

If the parameters of behaviors are unknown or time-varying, an activity model that can estimate parameters of the model "on the fly" is needed. We present an online BMS model for nonstationary behaviors. Assume that (A1)-A(3) and (A6) hold, and relax (A4)-(A5). The number of segments $N$ may be unknown, but (A6) can be used to restrict the complexity of $x(t)$ within a segment. This motivates the construction of a basis of behaviors. The basis elements represent generic primitives of motion depending upon the parameters of $A_{q(t)}$. Specifically, for the segment-wise linear dynamics of surveillance videos, we choose basis elements to model the following types of 2-D motion: straight line with constant velocity, straight line with constant acceleration, curved motion, start, and stop.

The eigenvalues of the system matrix $A$ are used to characterize the basis elements. Consider a linear time-invariant system $\dot{x}(t)=A x(t)$, where $A$ is a real-valued square matrix. Fixing the initial state $x(0)=x_{0}$, we have $x(t)=\exp (A t) x_{0}$, where $\exp (A t)=\sum_{k=0}^{\infty}\left(t^{k} / k !\right) A^{k}$ [30]. Depending on the eigenvalues $\lambda_{1}, \lambda_{2}$ of $A$, the equilibrium point exhibits the following types of behavior: curved trajectories (both eigenvalues are nonzero and real), straight line trajectories (one of the eigenvalues is zero), spiral trajectories (complex eigenvalues). These distinctions are syntactic rather than semantic, that is, these types of motion may be considered as a context-free vocabulary. We use these as the basis to describe behaviors of segments. Though the total number of behaviors may be unknown a priori, we can specify a basis of behaviors by partitioning the space of dynamics using the location of eigenvalues, that is, region in the space of allowable eigenvalues.

\section{APPROACH}

The estimation task in either offline or online BMS model consists of two main steps: computing the parameters of the behaviors, and identifying switching times between segments. It may be tempting to use the EM algorithm in this case [31]. The EM algorithm involves an iteration over the E-step to choose an optimal distribution over a fixed number of hidden states and the M-step to find the parameters of the distribution that maximize the data likelihood [31]. Unlike the classical HMM, however, the E-step is not tractable in switched-state space models [18]. To work around this, [18] presents a variational approach for estimating the parameters of switched-state space models, whereas [17] presents a sampling approach. Either of these approaches is applicable in the offline BMS case, but neither is suitable for the online BMS model. We propose an algorithm that has two main components: a basis of behaviors for approximating behaviors within segments and the Viterbi-based algorithm.

The parameters of each segment is chosen so that the approximation error $R\left(\tau, t_{0}, q\right)$ defined below is minimized:

$$
R\left(\tau, t_{0}, q\right)=\frac{1}{\tau-t_{0}} \int_{t_{0}}^{\tau}\left(x-\hat{x}_{q}\right)^{T}\left(x-\hat{x}_{q}\right) d t
$$

with $\dot{\hat{x}}_{q}(t)$ is a solution to $(9)$.

$R\left(\tau, t_{0}, q\right)$ is the accumulated cost of using the $q$ th family of behaviors to approximate the current segment. For linear dynamics, the least square estimate minimizes this error. This is consistent with the probability density estimates under normal assumption for AR-HMM.

\subsection{Viterbi-based algorithm}

The Viterbi algorithm is used to find the optimal state sequence $Q=\{q(1), q(2), \ldots, q(T)\}$ for the given observation sequence $X=\{x(1), x(2), \ldots, x(t)\}$, such that the joint probability of states and observation is maximized. To place the proposed Viterbi-based algorithm in context, we trace the modifications starting with the Viterbi algorithm for the classical HMM approach. The quantity $\delta(t, i)$ is defined as follows [29]:

$$
\delta(t, i)=\max _{q_{1}^{t-1}} f\left(q_{1}^{t-1}, q(t)=i, x_{1}^{t} \mid \lambda\right)
$$

where $\lambda$ is the given HMM or AR-HMM. In the classical 
HMM case, we assume a Markov state process $P\left(q(t) \mid q_{1}^{T}\right)=$ $P(q(t) \mid q(t-1))$ and that the observations are conditionally independent of the past given the current state, that is,

$$
f\left(x(t) \mid x_{1}^{T}, q_{q}^{T}\right)=f(x(t) \mid q(t)) .
$$

It allows us to express (11) recursively as follows:

$$
\delta(t, j)=\max _{1 \leq i \leq N}\left[\delta(t-1, i) a_{i j}\right] f(x(t) \mid q(t)=j),
$$

where $A=\left[a_{i j}\right]_{1 \leq i, j \leq N}$ is the state transition probability matrix. The $a_{i j}$ 's, which are stationary, can be estimated using the Baum-Welch algorithm (shown in the appendix). The trellis implementation of the Viterbi algorithm is used to compute the optimal state sequence efficiently. The size of the trellis is $N \times T$, where one observation variable $x(t)$ is involved at each stage [32]. In the AR-HMM, the observation probability equation is written as (4) instead of (12). It is easy to derive the optimal state sequence similar to the previous case. The major difference is that at each stage, the error computation involves a window of observed data $x_{t-\alpha-1}^{t-1}$ instead of one variable $x(t)$ [33].

Compared to AR-HMM, the offline BMS model is more general in that the evolution of state sequence is not Markov, but is allowed to depend on the continuous state (6). This makes the computation of joint probabilities for $\delta(t, i)$ difficult, as explained in Section 3.2. The effective state $r(t)=$ $\left(q(t), x_{t-2 \alpha}^{t-\alpha}\right)$, however, is Markov. We use this to set up a Viterbi-like algorithm based on approximation costs incurred in persisting in each behavior and switching cost due to transitions among behaviors. If the denominator in (6) could be computed, then these costs could be readily turned into probabilities. Also the probability $a_{i j}$ is not stationary anymore, and depends on the previous values of continuous state. The main difference in implementation is a reduced size of the trellis. By assumption (A2), the size of the trellis reduces from $N \times T$ to $N \times K$, where $K \alpha=T$ and $\alpha$ is the minimum size of each segment. This time axis is further halved due to effective state $r(t)$ being Markov instead of $q(t)$ as shown in (7) and (8). The recursive equations are given below. The online BMS case presents an additional challenge due to nonstationarity. In this case, the $N$ states represent $N$ basis elements of behaviors.

In (13), the basic principle of dynamic programming is used to write the recursive equation using two quantities: observation probability $f(x \mid q)$ and the state transition probability $a_{i j}$. The approximation cost $R\left(\tau, t_{0}, q\right)$ is an analog of $f(\cdot \mid \cdot)$. We define the switching cost to be an analog of $a_{i j}$. For the BMS model, the transition probability for the effective state is given in (7). Using (6), we have

$$
\begin{aligned}
f(q(t) & \left.=j \mid q(t-1)=i, x_{t-2 \alpha}^{t-\alpha}\right) \\
& =\frac{f\left(q(t)=j, q(t-1)=i \mid x_{t-2 \alpha}^{t-\alpha}\right)}{f\left(q(t-1)=i \mid x_{t-2 \alpha}^{t-\alpha}\right)} .
\end{aligned}
$$

Using (14), the switching cost $S: \partial \operatorname{Inv}(i) \times \partial \operatorname{Inv}(j) \rightarrow \mathcal{R}^{+}$ is defined as follows.
Let $t_{1} \in\left[\tau_{i}, \tau_{i+1}\right)$ be a candidate switching time. The larger the value of the switching function, the higher the error due to switching at $t_{1}$, that is, $\tau_{i+1}=t_{1}$, when the discrete state changes from $m$ to $n$. The invariant set $\operatorname{Inv}(i)$ denotes the continuous state dynamics for the hidden state $i$, that is, as long as $x(t) \in \operatorname{Inv}(i)$, we say that the object exhibits the behavior indexed by the index $i$. The boundary of the invariant set is denoted by $\partial \operatorname{Inv}(i)$,

$$
S(m, n)=\frac{\left(1+R\left(t_{1}, \tau_{i}, m\right)\right)\left(1+R\left(\tau_{i+1}, t_{1}, n\right)\right)}{\left(1+R\left(\tau_{i+1}, \tau_{i}, m\right)\right)} .
$$

The 1's are added to ensure that the function is well defined at all time instants. If $t_{1}$ was the true switching time, the approximation error in the numerator will be smaller than that in the denominator.

Let $\delta(k, n)$ denote the cost accumulated in the $n$th behavior at time $k$ and $\psi(k, n)$ represent the state at time $k$ which has the lowest cost corresponding to the transition to behavior $n$ at time $k$. The time index $k$ is used instead of $t$, to denote that switching is assumed to occur at discrete time instants (assumption (A2)).

(i) Initialization: for $n \in N$, let

$$
\begin{gathered}
\delta(1, n)=R(1,1, n), \\
\psi(1, n)=0 .
\end{gathered}
$$

(ii) Recursion: for $2 \leq 2 k \leq T$ and $1 \leq j \leq N$,

$$
\begin{gathered}
\delta(k, j)=\min _{1 \leq i \leq N}[\delta(k-1, i)-S(i, j)]-R\left(k, \tau_{k-1}, j\right), \\
\psi(k, j)=\arg \min _{1 \leq i \leq N}[\delta(k-1, i)-S(i, j)] .
\end{gathered}
$$

(iii) Termination:

$$
\begin{aligned}
C^{*} & =\min _{1 \leq i \leq N}[\delta(T, i)], \\
q^{*}(T) & =\arg \min _{1 \leq i \leq N}[\delta(T, i)] .
\end{aligned}
$$

(iv) Backtrack: for $k=T-1, \ldots, 1$,

$$
q^{*}(k)=\psi\left(k+1, q^{*}(k+1)\right)
$$

\subsection{Anomaly detection using offline BMS model}

It is common to have several examples of normal activities, and a very few samples of anomalies making it difficult to model anomalies. Therefore, anomaly detection can be formulated as change detection (or outlier detection) from the normal model. Anomalies can be either spatial, temporal, or both. Examples of anomalies are path violation, gaining unrestricted access, and so forth. Offline BMS models are trained using normal video sequences. Given a test (anomalous) video sequence, motion trajectories, and observation sequence are extracted as before. The Viterbi-based 


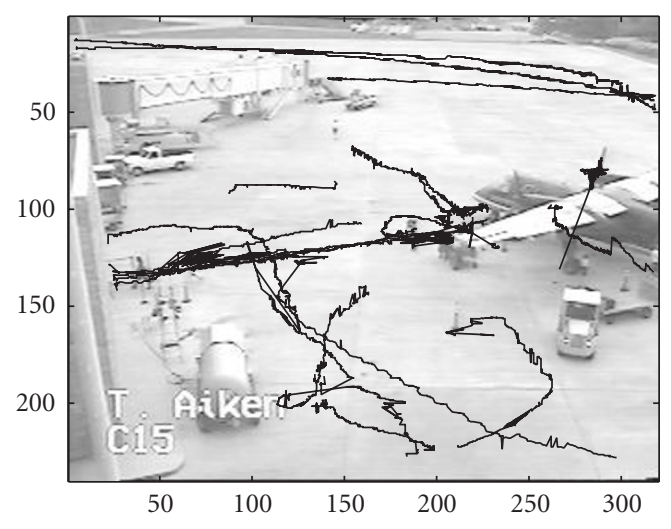

(a)

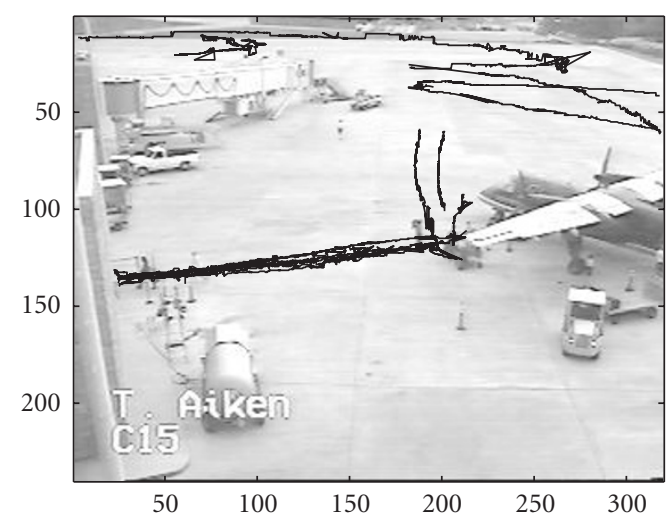

(b)

FIGURE 1: TSA airport tarmac surveillance dataset. Each image represents a block of 10000 frames along with motion trajectories extracted.

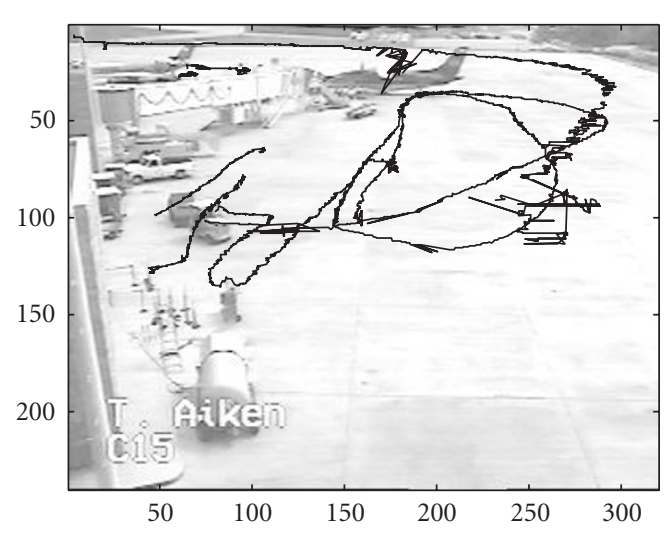

(a)

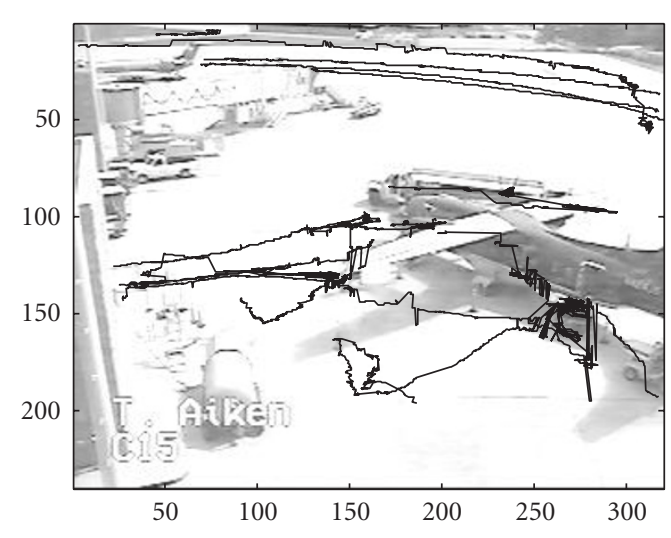

(b)

FIGURE 2: TSA airport tarmac surveillance dataset. Each image represents a block of 10000 frames along with motion trajectories extracted.

algorithm is initialized with parameters learnt using training data. If an unexpected state sequence is detected, an anomaly is declared. This assumes that short-time dynamics is consistent with the normal activity, but anomaly exists due to an unexpected sequencing. Thus a completely unrelated activity would not be declared an anomaly.

\section{EXPERIMENTS}

We demonstrate the usefulness of the online BMS model for temporal segmentation and the offline BMS model for anomaly detection using the following three datasets: the TSA airport surveillance dataset, bank dataset, and the UCF human action dataset.

\subsection{TSA airport surveillance dataset}

The TSA dataset consists of surveillance video captured at an airport tarmac [2]. The stationary camera operates at approximately 30 frames per second and the frame size is $320 \times 240$.
Though it is approximately 120 minutes long, a large portion of the video does not contain any activities. We divide the entire data into 23 blocks of about 10000 frames each. Here onwards, we refer to such sets of 10000 frames as blocks. Moving objects are detected and tracked as described in Section 2.1. The background at each pixel was modeled using a Gaussian distribution. The parameters are reinitialized every hundred frames. Each frame is compared with the background and the moving objects are detected. A bounding box is drawn on the detected blobs. The KLT algorithm is allowed to choose feature points for tracking within the bounding box. The average trajectory of feature points within the bounding box is regarded as the motion trajectory of the objects (Figures 1 and 2). Since the video sequence is long, it is impractical to obtain ground truth of trajectories. The activity model needs to be robust to imperfections in tracking. The ground truth for temporal segmentation was extracted manually, that is, by direct inspection of the video sequences.

In four blocks, we observe a significant amount of multiobject activity when planes arrive and depart. The four 
TABLE 1: TSA dataset: temporal segmentation of two blocks using the online BMS model. GCP = ground crew personnel, PAX = passengers, Det. $=$ segment detected, $\mathrm{TF}=$ tracking failed .

\begin{tabular}{l|c|c}
\hline Number & Block in Figure 1(a) & Comment \\
\hline 1 & 2 GCP split, walk away & Det. \\
2 & GCP across tarmac & Det. \\
3 & Truck arrives, GCP & Det. \\
4 & GCP across tarmac & Det. \\
5 & Truck & Det. \\
6 & GCP movement & Det. \\
7 & Plane-I arrives & Det. \\
8 & Luggage cart to plane & Det. \\
9 & Truck crossing scene & TF \\
& Plane-II arrives & Det. \\
10 & GCP and luggage cart & - \\
11 & approach plane-I & Det. \\
12 & PAX disembark & Extra segment \\
\hline
\end{tabular}

TABLE 2: TSA dataset: temporal segmentation of two blocks using the online BMS model. GCP = ground crew personnel, PAX = passengers, Det. $=$ segment detected, $\mathrm{TF}=$ tracking failed.

\begin{tabular}{l|c|c}
\hline Number & Block in Figure 1(b) & Comment \\
\hline 1 & Luggage cart & TF. \\
2 & GCP movement near plane & Det. \\
3 & Luggage cart enter & Det. \\
4 & Plane enters & Det. \\
5 & PAX embark & Det. \\
6 & Luggage cart & TF. \\
7 & PAX embark & Det. \\
\hline
\end{tabular}

blocks form the test set. Figures 1 and 2 show the motion trajectories for these blocks. The remaining portion of the dataset is used as the training set. It may seem large compared to the size of the test set. The activity content, however, is not as dense as in the test set. The paucity of training data makes it unrealistic to train a model in the conventional sense, where parameters of the mixed-state model are estimated. Instead, we train an online BMS model, which involves finding a basis of behavior. The values of parameters are less important than the region of parameter space they represent. Accordingly, the basis has elements that can produce the following types of motion: constant velocity along a straight line, constant acceleration along a straight line, curved trajectories with constant velocity, start, and stop.

We demonstrate temporal segmentation of the four test blocks using the online BMS model. The segmentation results for the four blocks shown in Figures 1(a)-1(b) and 2(a)2 (b) are summarized in Tables 1-4, respectively. On an average, there were $15 \%$ missed detections in segmentation. This was mainly because of tracking errors.
TABLE 3: TSA dataset: temporal segmentation of two blocks using the online BMS model. GCP = ground crew personnel, PAX = passengers, Det. $=$ segment detected.

\begin{tabular}{l|c|c}
\hline Number & Block in Figure 2(a) & Comment \\
\hline 1 & Plane exits & Det. \\
2 & GCP movement & Det. \\
3 & Luggage cart to plane-II & Det. \\
4 & Bag falls off luggage cart & Det. \\
5 & Luggage cart to plane-II & Det. \\
\hline
\end{tabular}

TABLE 4: TSA dataset: temporal segmentation of two blocks using the online BMS model. GCP = ground crew personnel, PAX = passengers, Det. $=$ segment detected, $\mathrm{TF}=$ tracking failed .

\begin{tabular}{c|c|c}
\hline Number & Block in Figure 2(b) & Comment \\
\hline 1 & 2 GCP movement & Det. \\
2 & Luggage cart & TF \\
3 & GCP movement & TF \\
4 & Plane-II arrives & Det. \\
5 & GCP movement & TF \\
6 & Luggage cart to plane-II & Det. \\
7 & Plane-III arrives & Det. \\
8 & PAX embark & Det. \\
9 & Truck movement & Det. \\
10 & GCP near plane-II & TF \\
11 & Luggage cart from plane-II & Det. \\
12 & More PAX embark & Det. \\
\hline
\end{tabular}

\subsection{Bank surveillance dataset}

The bank dataset consists of staged videos collected at a bank [34]. There are four sequences, each approximately 15-20 seconds ( $\sim 400$ frames) long. Figures 3 and 4 show sample images from the dataset. The actors demonstrate two types of scenarios.

(i) Attack scenario where a subject coming into the bank forces his way into the restricted area. This is considered as an anomaly.

(ii) No attack scenario where subjects enter/exit the bank and conduct normal transactions. This depicts a normal scenario. The normal process of transactions is known a priori and we train an offline BMS model using these trajectories.

\subsubsection{Temporal segmentation}

We retained the same basis of behavior that was used for the TSA dataset in Section 5.1. Though the TSA data is captured outdoors and the bank data indoors, they are both surveillance videos. They retain similarity at the primitive or behavior level. For the no attack scenario, segmentation using the online BMS model yielded two parts. In the first segment, we see two subjects entering the bank successively. The first person goes to the paper slips area and the second person goes to 


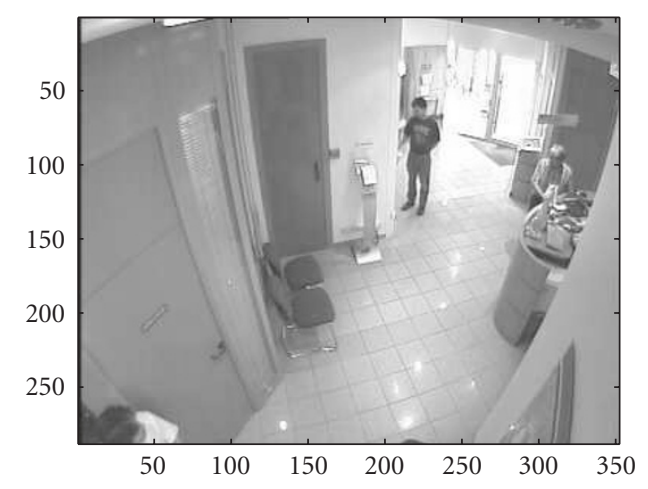

(a)

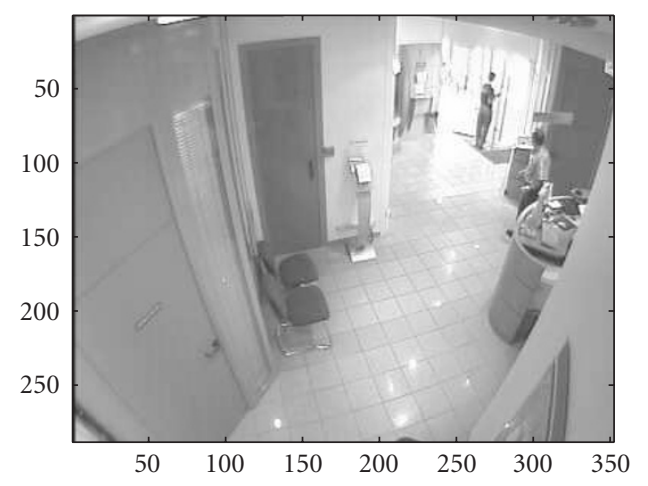

(b)

FIgURE 3: Bank dataset: two segments detected in the no attack scenario: (a) a subject enters the bank, goes to the area where paper slips are stored. Another subject enters the bank and goes to the counter area, (b) exit bank.

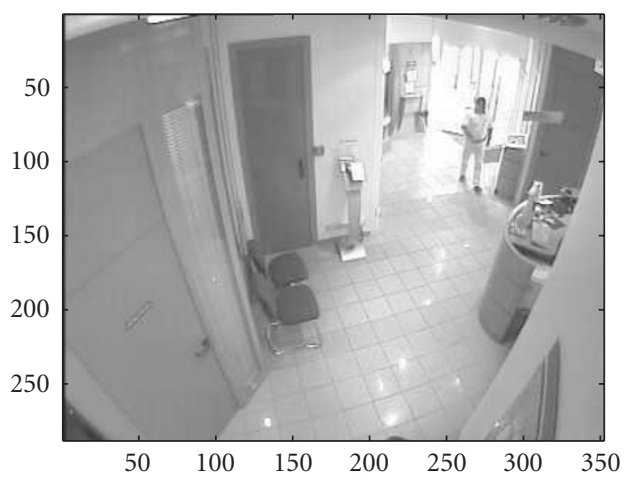

(a)

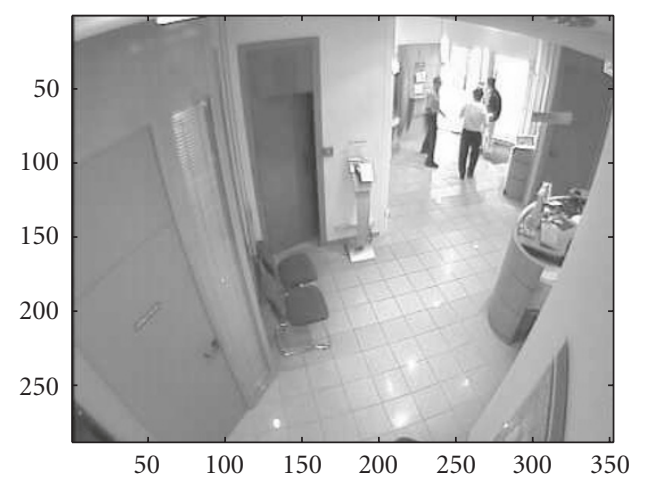

(c)

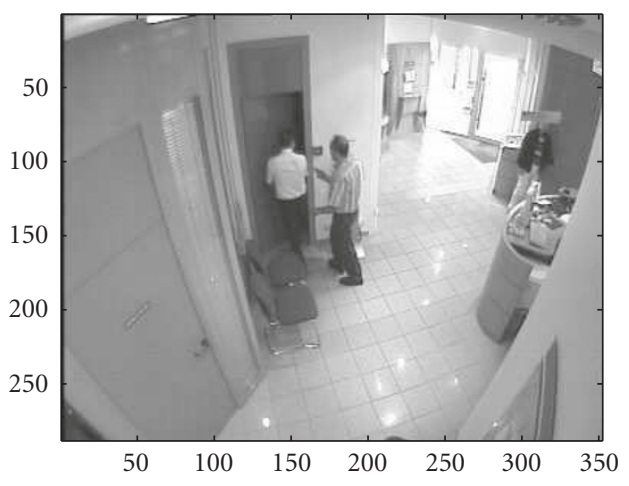

(b)

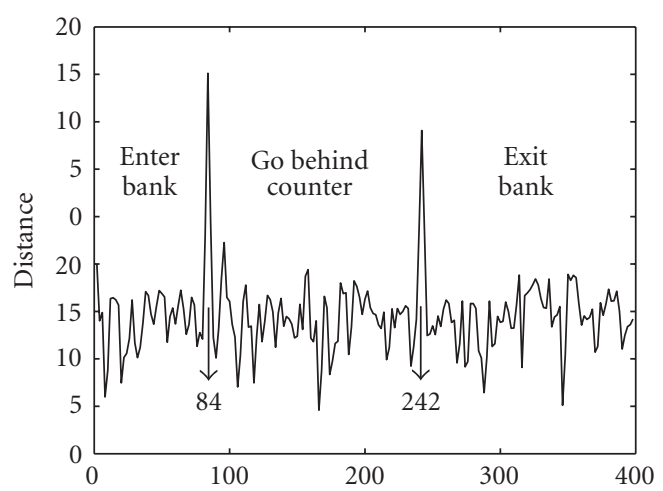

(d)

FIgURE 4: Bank dataset: three segments detected in the attack scenario: (a) enter bank, (b) gain access to the restricted area behind the counter, and (c) exit bank. (d) shows a plot of the switching function. Peaks in the plot indicate boundaries in temporal segmentation.

the counter. In the second segment, the two subjects leave the bank. Figure 3 shows sample images from the two segments. We store the parameters of these behavioral segments as the normal activity.

Figure 4 shows an example of an attack scenario. Here, the online BSM model yielded three segments. In the first segment, the person enters the bank and proceeds to the area where the deposit/withdrawal slips are kept. This is similar to the first segment in the no attack case. During the second segment, he follows another person into the restricted area behind the counter. The third segment consists of the person leaving the bank. 
TABLE 5: Comparing no attack and attack scenarios in bank surveillance data. $L 1$ distance between histograms of parameters of online BMS model is used as similarity score.

\begin{tabular}{l|c|c|c|c}
\hline Number & No attack & Attack 1 & Attack 2 & Attack 3 \\
\hline No attack & 0 & 310 & 424 & 362 \\
Attack 1 & 310 & 0 & 218 & 278 \\
Attack 2 & 424 & 218 & 0 & 180 \\
Attack 3 & 362 & 278 & 180 & 0 \\
\hline
\end{tabular}

\subsubsection{Anomaly detection}

The parameters of an offline BMS model are estimated using the no attack scenario. To detect the presence of an anomaly, we compute the error accumulated along the optimal state sequence using the test trajectory. It is difficult to assess the performance of this naive scheme since we have very few samples. Alternatively, we use the online BMS model to detect anomalies. If we assume that the attack scenarios were normal activities while the no attack scenario was an anomaly, we may expect the comparison scores of the different attack scenarios to be clustered together. For each of the four scenarios in the dataset, parameters of their online BMS models are computed. We form a similarity matrix of size $4 \times 4$ in order to check whether the attack scenarios cluster separately. The $L 1$ distance between the histograms of parameters of learnt behavior is used as the similarity score. Table 5 shows the distance between the different attack examples with the no attack case. We observe that the attack scenarios are more similar to each other compared to the no attack scenario.

\subsubsection{Comparison of results}

Georis et al. [34] presented an ontology-based approach for video interpretation in which activities of interest are manually encoded. They demonstrated the effectiveness of ontologies for detecting attacks on a safe in a bank monitoring dataset. Their method requires a detailed description in the form of a set of rules to detect an "attack" activity. The proposed method, however, is data driven. The extent of deviation observed in a given video sequence compared to a normal scenario is used as a measure for detecting anomalies. Comparitive results are summarized below.

In [34], the authors report the following results on tracking persons in the bank scene: $88 \%$ true positives, $12 \%$ false negatives, and $2 \%$ false positives. There were no errors in tracking in our method.

For anomaly detection (i.e., detecting that the bank safe was attacked), the results reported in [34] are $93.5 \%$ of true positives, $6.25 \%$ of false negatives, and $0 \%$ of false positives. These results correspond to 16 repetitions of the attack scenario. We have access to only 3 attack scenarios. On these, we obtained correct anomaly detection in all three scenarios.

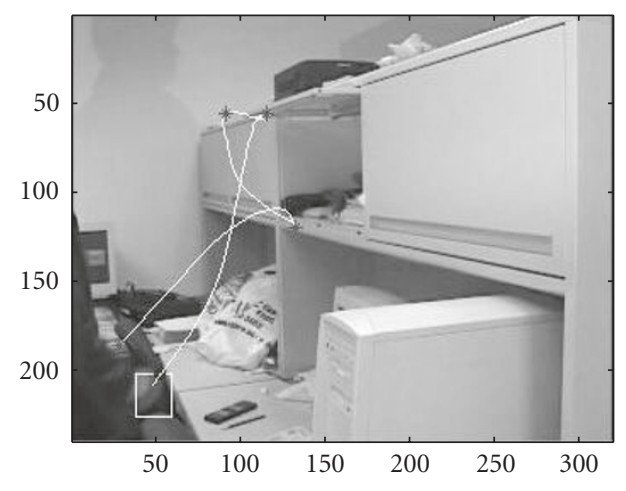

(a)

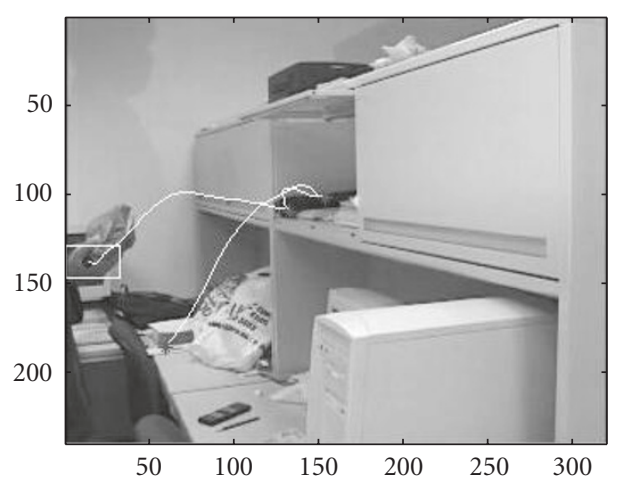

(b)

Figure 5: Sample images from UCF dataset.

\subsection{UCF human action dataset}

We may think of many actions as a sequence of behaviors. For example, picking up an object may be abstracted as extend the hand toward object-grab object-withdraw the hand; erasing the black board, as extend hand-move hand side to side on the board-withdraw hand; opening the door, as extend hand-grab knob-withdraw hand. To generate an action, we may compose a sequence of systems that operate with the appropriate parameters.

The UCF database of human actions consists of 60 video sequences captured in an office environment [13]. Examples of actions include picking up an object, putting down an object, opening the cabinet door, and pouring water into a cup. A brief description of the low-level video processing algorithms for extracting trajectories is given below. Further details are available in [13]. The dataset obtained from the UCF group contains the extracted trajectories. The hand was detected using a skin-detection algorithm. A mean-shift tracker was initialized at the detected position to obtain motion trajectory of the hand. The trajectories were smoothed out using anisotropic diffusion. Figure 5 shows sample images from the database along with extracted motion trajectories.

We employ the Viterbi-based segmentation described in Section 4.1 to find the segments of actions. We show some 


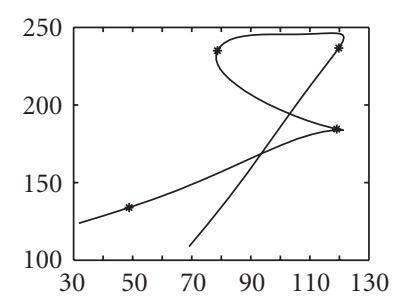

(a)

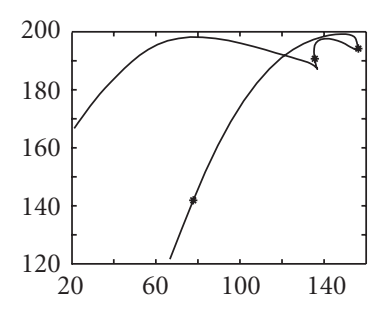

(e)

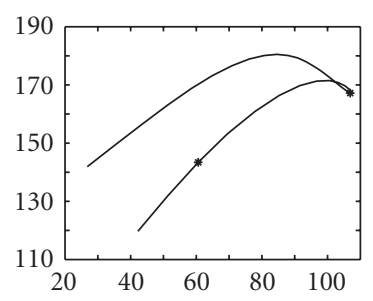

(i)

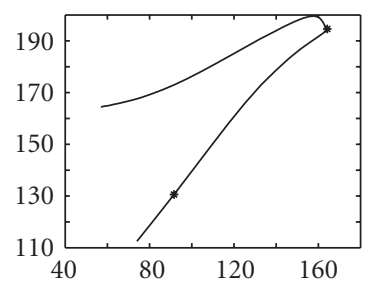

(b)

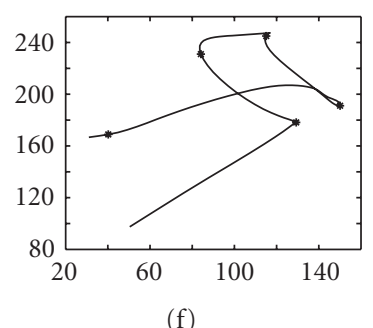

(f)

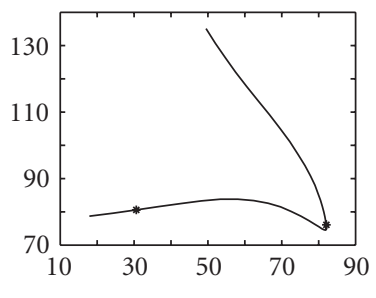

(j)

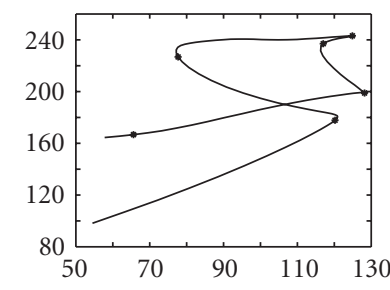

(c)

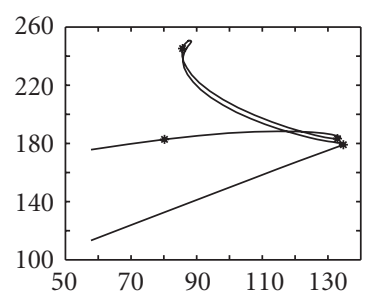

(g)

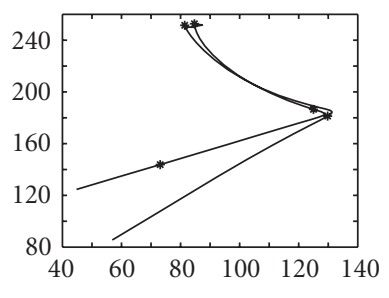

(k)

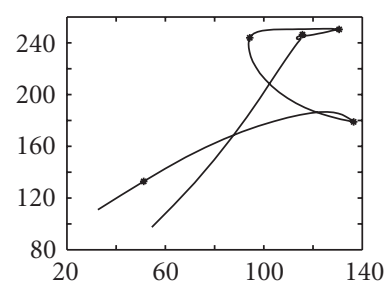

(d)

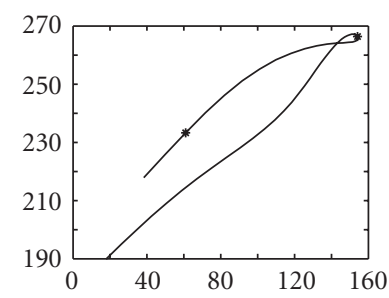

(h)

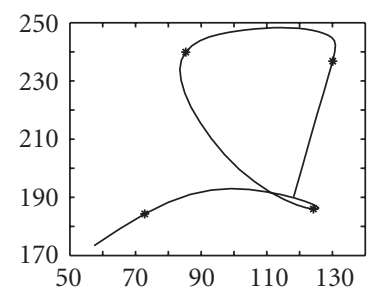

(1)

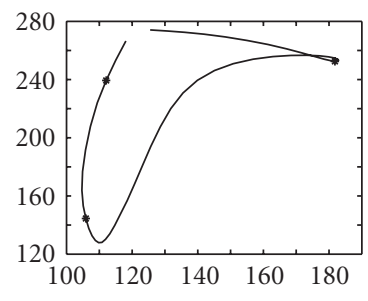

(m)

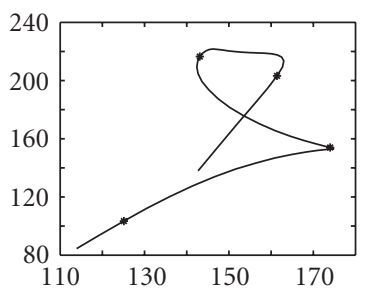

(n)

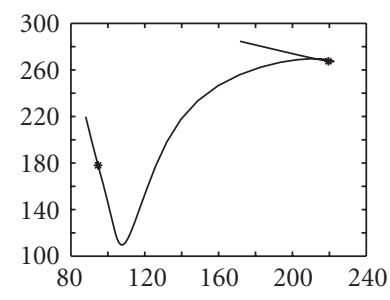

(o)

FIGURE 6: Motion trajectories of the hand in the UCF dataset for different actions. The dots along the trajectory denote segment boundaries detected.

of the segmentation results in Figure 6. A description of motion trajectories shown in Figure 6 along with the detected segment boundaries is given below.

(a) Open cabinet door: start-reach door handle-open doorwithdraw hand.

(b) Pick up object: start-pick up object.

(c) Put down: start-put object in cabinet-reach door handleclose door-withdraw hand.

(d) Open cabinet door: start-reach door handle-open doorextra segment-withdraw hand.

(e) Pick up object: start-pick up object.

(f) Put down: start-put object in cabinet-reach door handleclose door-withdraw hand.

(g) Open cabinet door: start-reach door handle-open doorwithdraw hand. (h), (i), (j) Pick up object: start-pick up object.

(k), (l) Open cabinet door: start-reach door handle-open door-withdraw hand.

(m) Pick up and put down elsewhere: pick up-put downwithdraw hand

(n) Open cabinet door (different viewing direction): startreach door handle-open door-withdraw hand.

(o) Pick up and put down elsewhere: pick up-put down (late detection)-withdraw hand.

Table 6 shows the mean and variance of the number of segments detected for different activities. The same number of segments were detected consistently across multiple samples of activities picking up and pouring water an object. On the other hand, variance in the number of segments detected for picking up an object and putting it down elsewhere was 
TABle 6: Number of segments detected for activities in the UCF indoor human action dataset.

\begin{tabular}{lcc}
\hline Activity & $\begin{array}{c}\text { Average no. of } \\
\text { segments }\end{array}$ & Variance \\
\hline Open door & 4.44 & 0.53 \\
Pick up & 2.27 & 0.21 \\
Put down & 2.50 & 0.94 \\
Close door & 4.25 & 0.25 \\
Erase & 6.50 & 0.33 \\
Pour water & 3.00 & 0.00 \\
Pick up object and & 3.75 & 3.92 \\
put down elsewhere & & \\
\hline
\end{tabular}

high. This was because of excessive differences in appearance across samples.

\subsubsection{Comparison of results}

We compared the location of detected segment boundaries with the dynamic instants described in [13]. Dynamic instants are points of high curvature along the trajectory. They are chosen as the feature of interest to ensure view invariant representation of actions. The first segment switching in the proposed approach occurs after sufficient evidence about the dynamics has been accumulated. This is marked as the start segment, which usually occurs in 10-15 frames. We ignore this boundary point when comparing our results with dynamic instants in [13] since it does not have an explicit start instant. Segment boundaries detected using our method, which are marked by dots in Figure 6 are compared with the dynamic instants in [13]. The average difference between the two values are as follows: 5.4 frames for open door, 4.1 frames for close door, 3.3 frames for pour water, 2.0 frames for erase board, and 6.2 frames for pick up object and put down elsewhere.

\subsection{Homecare applications}

Though the proposed approach was demonstrated using video sequences collected in office and airport environments, it can be easily applied to home-care scenarios. DeNatale et al. [35] describe fall detection and accessing unauthorized locations as typical applications in home-care scenarios. We highlight the applicability of our method to some home-care applications.

As demonstrated using the bank monitoring dataset, our approach is able to detect if a person gained access to unauthorized places. Similarly, as experiments using the UCF dataset demonstrated, human actions were segmented into a sequence of elementary parts. For instance, opening a cabinet door was represented as start-reach door handle-open doorwithdraw hand. These actions could be used to find the number of times medicine cabinet is used. If an opening action does not occur at expected times, an alarm could be issued.

\section{SUMMARY}

It is important to build activity models that generalize across scenarios so that they enhance portability and adaptability. Even within a scenario, it may not be realistic to enumerate all possible activities during the design process. Instead, the system should be capable of learning new behavioral models. Approaches that are completely data driven may not be ideal since combinatorially many alternatives have to be considered.

To summarize, we have described piecewise linear mixedstate models for representing activities using motion trajectories as observed data. A sequence of such segments is said to characterize an activity based on a context-independent basis of behavior. Parameters of the segmentwise models and switching times between them were estimated using a Viterbi-based algorithm. Experiments using surveillance video streams in both indoor and outdoor settings demonstrate that the method can be used to analyze activities at different scales. The usefulness of the proposed method is shown using applications such as temporal segmentation and anomaly detection. As part of future work, we will investigate ways to learn the basis elements from training sequences.

\section{APPENDIX}

\section{BAUM-WELCH ALGORITHM}

This section contains a brief overview of the Baum-Welch algorithm that was introduced in [31]. There are several sources that offer a detailed explanation (e.g., $[29,31])$. Let $\lambda=(A, B, \Pi)$ represent an HMM, where $A=\left[a_{i j}\right]$ is the transition probability matrix, $B$ contains emission probabilities conditioned on the current state, and $\Pi$ is the initial distribution of states. Let $O=\left\{o_{1}, o_{2}, \ldots, o_{T}\right\}$ be the observation sequence. The Baum-Welch algorithm is an expectationmaximization (EM) algorithm that can compute parameters of $A, B$, and $\Pi$ such that the likelihood function $P(O \mid \lambda)$ is maximized. It involves the following steps.

(i) Choose the initial parameters of $\lambda$ (usually through a $k$-means procedure).

(ii) Reestimate the parameters using the forward and backward variables. This is equivalent to computing estimates such that

$$
\begin{gathered}
\bar{a}_{i j}=\frac{\text { expected no. of transitions from state } i \text { to } j}{\text { expected no. of transitions from state } i}, \\
\bar{b}_{j}(o)=\frac{\text { expected no. of times in state } j \text { and observing } o}{\text { expected no. of times in state } j} .
\end{gathered}
$$

(iii) Iterate until convergence. 


\section{ACKNOWLEDGMENT}

This work was partially supported by the ARDA/VACE Program under the Contract 2004H80200000.

\section{REFERENCES}

[1] B. Sin and J. H. Kim, "Nonstationary hidden Markov model," Signal Processing, vol. 46, no. 1, pp. 31-46, 1995.

[2] N. Vaswani, A. R. Chowdhury, and R. Chellappa, "Activity recognition using the dynamics of the configuration of interacting objects," in Proceedings of the IEEE Computer Society Conference on Computer Vision and Pattern Recognition (CVPR '03), vol. 2, pp. 633-640, Madison, Wis, USA, June 2003.

[3] J. K. Aggarwal and Q. Cai, "Human motion analysis: a review," Computer Vision and Image Understanding, vol. 73, no. 3, pp. 428-440, 1999.

[4] T. Starner and A. Pentland, "Real-time American Sign Language recognition from video using hidden Markov models," in Proceedings of the IEEE International Symposium on Computer Vision (ISCV'95), pp. 265-270, Coral Gables, Fla, USA, November 1995.

[5] R. Polana and R. Nelson, "Low level recognition of human motion (or how to get your man without finding his body parts)," in Proceedings of the IEEE Workshop on Motion of NonRigid and Articulated Objects, pp. 77-82, Austin, Tex, USA, November 1994.

[6] A. Bobick and J. Davis, "Real-time recognition of activity using temporal templates," in Proceedings of the 3rd IEEE Workshop on Applications of Computer Vision (WACV'96), pp. 3942, Sarasota, Fla, USA, December 1996.

[7] Y. A. Ivanov and A. F. Bobick, "Recognition of visual activities and interactions by stochastic parsing," IEEE Transactions on Pattern Analysis and Machine Intelligence, vol. 22, no. 8, pp. 852-872, 2000.

[8] A. Kale, A. Sundaresan, A. N. Rajagopalan, et al., "Identification of humans using gait," IEEE Transactions on Image Processing, vol. 13, no. 9, pp. 1163-1173, 2004.

[9] T. Izo and W. E. L. Grimson, "Simultaneous pose estimation and camera calibration from multiple views," in Proceedings of IEEE Workshop on Motion of Non-Rigid and Articulated Objects, vol. 1, pp. 14-21, Washington, DC, USA, June 2004.

[10] M. Brand, N. Oliver, and A. Pentland, "Coupled hidden Markov models for complex action recognition," in Proceedings of the IEEE Computer Society Conference on Computer Vision and Pattern Recognition (CVPR '97), pp. 994-999, San Juan, Puerto Rico, USA, June 1997.

[11] R. Nevatia, T. Zhao, and S. Hongeng, "Hierarchical languagebased representation of events in video streams," in Proceedings of 2nd IEEE Workshop on Event Mining: Detection and Recognition of Events in Video, vol. 4, pp. 39-45, Madison, Wis, USA, June 2003.

[12] T. Syeda-Mahmood, A. Vasilescu, and S. Sethi, "Recognizing action events from multiple viewpoints," in Proceedings of IEEE Workshop on Detection and Recognition of Events in Video, Vancouver, Canada, July 2001.

[13] C. Rao, A. Yilmaz, and M. Shah, "View-invariant representation and recognition of actions," International Journal of Computer Vision, vol. 50, no. 2, pp. 203-226, 2002.

[14] D. Koller and U. Lerner, "Sampling in factored dynamic systems," in Sequential Monte Carlo Methods in Practice, pp. 445464, Springer, New York, NY, USA, 2001.
[15] R. Hamid, Y. Huang, and I. Essa, "ARGMode-activity recognition using graphical models," in Proceedings of IEEE Computer Society Conference on Computer Vision and Pattern Recognition (CVPR '03), vol. 4, pp. 38-43, Madison, Wis, USA, June 2003

[16] V. Vu, F. Bremond, and M. Thonnat, "Automatic video interpretation: a novel algorithm for temporal scenario recognition," in Proceedings of the 18th International Joint Conferences on Artificial Intelligence (IJCAI '03), Acapulco, Mexico, August 2003.

[17] M. Isard and A. Blake, "A mixed-state condensation tracker with automatic model-switching," in Proceedings of the 6th IEEE International Conference on Computer Vision (ICCV'98), pp. 107-112, Bombay, India, January 1998.

[18] Z. Ghahramani and G. E. Hinton, "Variational learning for switching state-space models," Neural Computation, vol. 12, no. 4, pp. 831-864, 2000.

[19] A. B. Kurzhanski and P. Varaiya, "Dynamic optimization for reachability problems," Journal of Optimization Theory and Applications, vol. 108, no. 2, pp. 227-251, 2001.

[20] C. Tomlin, G. J. Pappas, and S. Sastry, "Conflict resolution for air traffic management: a study in multiagent hybrid systems," IEEE Transactions on Automatic Control, vol. 43, no. 4, pp. 509-521, 1998.

[21] C. Stauffer and W. E. L. Grimson, "Learning patterns of activity using real-time tracking," IEEE Transactions on Pattern Analysis and Machine Intelligence, vol. 22, no. 8, pp. 747-757, 2000.

[22] V. Parameswaran and R. Chellappa, "View invariance for human action recognition," International Journal of Computer Vision, vol. 66, no. 1, pp. 83-101, 2006.

[23] D. G. Kendall, D. Barden, T. K. Carne, and H. Le, Shape and Shape Theory, John Wiley \& Sons, New York, NY, USA, 1999.

[24] H. Zhong, J. Shi, and M. Visontai, "Detecting unusual activity in video," in Proceedings of the IEEE Computer Society Conference on Computer Vision and Pattern Recognition (CVPR '04), vol. 2, pp. 819-826, Washington, DC, USA, June 2004.

[25] I. Haritaoglu, R. Cutler, D. Harwood, and L. S. Davis, "Backpack: detection of people carrying objects using silhouettes," in Proceedings of the 7th IEEE International Conference on Computer Vision (ICCV '99), vol. 1, pp. 102-107, Kerkyra, Greece, September 1999.

[26] C. R. Wren, A. Azarbayejani, T. Darrell, and A. P. Pentland, "Pfinder: real-time tracking of the human body," IEEE Transactions on Pattern Analysis and Machine Intelligence, vol. 19, no. 7, pp. 780-785, 1997.

[27] B. D. Lucas and T. Kanade, "An iterative image registration technique with an application to stereo vision," in Proceedings of the 7th International Joint Conference on Artificial Intelligence (IJCAI '81), pp. 674-679, Vancouver, BC, Canada, August 1981.

[28] Y. Ephraim, A. Dembo, and L. R. Rabiner, "Minimum discrimination information approach for hidden Markov modeling," IEEE Transactions on Information Theory, vol. 35, no. 5, pp. 1001-1013, 1989.

[29] L. R. Rabiner, "A tutorial on hidden Markov models and selected applications in speech recognition," Proceedings of the IEEE, vol. 77, no. 2, pp. 257-286, 1989.

[30] M. Vidyasagar, Nonlinear Systems Analysis, Prentice Hall, Englewood Cliffs, NJ, USA, 1993.

[31] L. Baum, T. Petrie, G. Soules, and N. Weiss, "A maximization technique occuring in the statistical analysis of probabilistic functions of Markov chains," The Annals of Mathematical Statistics, vol. 41, no. 1, pp. 164-171, 1970. 
[32] G. D. Forney Jr., "The Viterbi algorithm," Proceedings of the IEEE, vol. 61, no. 3, pp. 268-278, 1973.

[33] A. Kavčić and J. M. F. Moura, "The Viterbi algorithm and Markov noise memory," IEEE Transactions on Information Theory, vol. 46, no. 1, pp. 291-301, 2000.

[34] B. Georis, M. Maziere, F. Bremond, and M. Thonnat, "A video interpretation platform applied to bank agency monitoring," in Proceedings of Workshop on Intelligent Distributed Surveillance Systems (IDSS '04), pp. 46-50, London, UK, February 2004.

[35] F. DeNatale, O. Mayora-Ibarra, and L. Prisciandaro, "Interactive home assistant for supporting elderly citizens," in Proceedings of EUSAI Workshop on Ambient Intelligence Technologies for WellBeing at Home, Eindhoven, The Netherlands, November 2004.

Naresh P. Cuntoor received the B.E. degree in electronics and communication engineering from the Karnataka Regional Engineering College (now renamed National Institute of Technology), Surathkal, India, in 2000, and the M.S. degree in electrical and computer engineering from the University of Maryland, College Park, in 2003, where he is currently pursuing the Ph.D. degree in electrical and computer engineering.

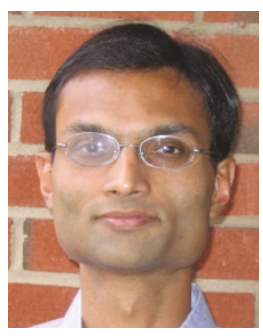
His research interests include computer vision, statistical pattern recognition, image processing, differential geometry, and topology.

Rama Chellappa received the B.E.(Hons) degree from the University of Madras, India, in 1975, and the M.E.(Distinction) degree from the Indian Institute of Science, Bangalore, in 1977. He received the M.S.E.E. and Ph.D. degrees in electrical engineering from Purdue University, West Lafayette, IN, in 1978 and 1981, respectively. Since 1991, he has been a Professor of electrical engineering and an Affiliate Pro-

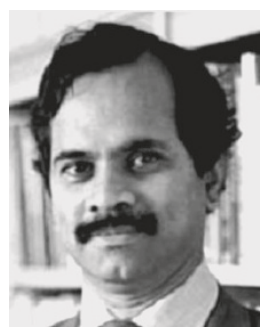
fessor of Computer Science at the University of Maryland, College Park. Recently, he was named the Minta Martin Professor of Engineering. He is also affiliated with the Center for Automation Research (Director) and the Institute for Advanced Computer Studies (PermanentMember). Prior to joining the University of Maryland, he was an Assistant Professor (1981-1986) and an Associate Professor (1986-1991) and Director of the Signal and Image Processing Institute (1988 to 1990) with the University of Southern California (USC), Los Angeles. Over the last 25 years, he has published numerous book chapters and peer-reviewed journal and conference papers. He has also coedited and coauthored many research monographs. His current research interests are face and gait analysis, 3D modeling from video, automatic target recognition from stationary andmoving platforms, surveillance and monitoring, hyperspectral processing, image understanding, and commercial applications of image processing and understanding. 